\title{
छூ \\ High quality electron bunch generation using a longitudinal density-tailored plasma-based accelerator in the three-dimensional blowout regime
}

\author{
X. L. Xu, ${ }^{1,{ }^{*}}$ F. Li, ${ }^{2}$ W. An, ${ }^{1}$ T. N. Dalichaouch, ${ }^{1}$ P. Yu, ${ }^{1}$ W. Lu, ${ }^{2,3, \dagger}$ C. Joshi, ${ }^{1}$ and W. B. Mori ${ }^{1}$ \\ ${ }^{1}$ University of California, Los Angeles, California 90095, USA \\ ${ }^{2}$ Department of Engineering Physics, Tsinghua University, Beijing 100084, China \\ ${ }^{3}$ IFSA Collaborative Innovation Center, Shanghai Jiao Tong University, Shanghai 200240, China
}

(Received 4 October 2016; published 21 November 2017)

\begin{abstract}
The generation of very high quality electron bunches (high brightness and low energy spread) from a plasma-based accelerator in the three-dimensional blowout regime using self-injection in tailored plasma density profiles is analyzed theoretically and with particle-in-cell simulations. The underlying physical mechanism that leads to the generation of high quality electrons is uncovered by tracking the trajectories of the electrons in the sheath that are trapped by the wake. Details on how the intensity of the driver and the density scale-length of the plasma control the ultimate beam quality are described. Three-dimensional particle-in-cell simulations indicate that this concept has the potential to produce beams with peak brightnesses between $10^{20}$ and $10^{21} \mathrm{~A} / \mathrm{m}^{2} / \mathrm{rad}^{2}$ and with absolute slice energy spreads of $\sim O(0.1) \mathrm{MeV}$ using existing lasers or electron beams to drive nonlinear wakefields. We also show projected energy spreads as low as $\sim 0.3 \mathrm{MeV}$ for half the charge can be generated at an optimized acceleration distance.
\end{abstract}

DOI: 10.1103/PhysRevAccelBeams.20.111303

Research in plasma-based acceleration (PBA) driven by a laser pulse or a relativistic electron beam is very active [1] because the large accelerating gradients in plasma wave wakefields may lead to compact accelerators. PBA is also capable of self-generating electron bunches that have significant charge $(\mathrm{Q})$, short duration $(\tau)$ and low normalized emittance $\left(\epsilon_{n}\right)$. A combination of these quantities define the normalized beam brightness $B_{n}=2 I / \epsilon_{n}^{2}$ where $I=Q / \tau$ is the current. While PBA experiments have produced useful beams, they have not produced beams with the necessary brightness and energy spread needed to drive an $\mathrm{x}$-ray free-electron-laser (X-FEL) [2] or the charge and emittance needed as an injector for a future linear collider [3].

The electron bunches needed to load plasma wakefields are very short and need to be synchronized with the driver. Therefore, self-injection has been actively investigated. The threshold for self-injection of electrons into nonlinear three-dimensional (3D) plasma waves in uniform plasmas has been studied in simulations and experiments [4-8]. Even in simulations, this process does not appear to be capable of generating the high quality beams needed for $\mathrm{X}$-FELs or a linear collider [9-11]. Therefore there has

\footnotetext{
*xinluxu11@ucla.edu

weilu@mail.tsinghua.edu.cn
}

Published by the American Physical Society under the terms of the Creative Commons Attribution 4.0 International license. Further distribution of this work must maintain attribution to the author(s) and the published article's title, journal citation, and DOI. been much recent work on methods for generating high brightness beams through controlled injection. These ideas fall into three categories. In one, electrons are born inside the wake through field ionization where the wake potential is near a maximum which eases the trapping threshold [12-14]. There are now numerous variations of this idea in which the injection and wake excitation are separated [15-17]. In the second, one or more laser pulses are used to trigger injection inside one plasma wake bucket [18-21]. In the third, which we consider here, the effective phase velocity of the wake is slowed down either by a density transition from high to low density [22,23], or through an expanding bubble from the evolution of a laser driver $[4,7,24,25]$.

In this article, we show that under appropriate conditions density downramp injection (Fig. 1) in the 3D blowout regime can generate unprecedented brightnesses $\left(10^{20}-10^{21} \mathrm{~A} / \mathrm{m}^{2} / \mathrm{rad}^{2}\right)$ and low slice energy spreads of $\sim O(0.1) \mathrm{MeV}$. In addition we show that low projected energy spread of half the beam charge can be achieved by optimizing the acceleration length. None of the ionization based injection schemes nor previous work on downramp injection has indicated that such beam parameters can be produced.

As first pointed out by Katsouleas [26], the phase velocity of a wake driven by a particle beam moving with a constant velocity $v_{d}$ in a density gradient will change due to the density dependence of the oscillation frequency. The phase of the plasma wave wake behind the driver can be written as $\phi(z, t)=\omega_{p}(z)\left(z / v_{d}-t\right)$, where $\omega_{p}(z)$ is the local plasma frequency. Thus the phase velocity of the wake is 


$$
v_{\phi}(z, t)=\frac{v_{d}}{1-\left(\mathrm{d} \omega_{p} / \mathrm{d} z\right) \omega_{p}^{-1}\left(v_{d} t-z\right)}
$$

Thus a density gradient can be used to increase the phase velocity (upramp) or decrease the phase velocity (downramp). The concept of using variations of the plasma density to trigger injection was proposed in gradual [22] and sudden [23] density transitions from a high density plasma to a low density plasma. These analyses were based on 1D arguments and paid little attention to the beam quality. There have been some recent results based on multidimensional simulations [27-30], but not for the parameters needed to observe the high quality beam generation described here.

In this article, we analyze the self-injection in density downramps from wakes excited in the nonlinear blowout regime using theory and 3D OSIRIS [31] simulations. We find that unprecedented brightnesses are generated due to the discovery that in the rear of the bubble the electrons experience defocusing fields that reduce their transverse momentum just as they are becoming trapped and which vanish after they are trapped. This process also leads to extremely low absolute slice energy spreads because of the mapping between the initial position of the particle and its location in the axial direction when it is trapped and extremely low absolute projected energy spread due to the combination of the injection and the following acceleration.

The processes behind the injection and the role of the defocusing fields on the generation of ultra bright electron beams are clearly illuminated by tracking particles of interest. To isolate the physics we use a nonevolving ultrarelativistic electron beam to produce the wake; however, when evolving beams or lasers are used similar results are obtained. The phase velocity is controlled by the density dependence of the blowout radius, so by adjusting the magnitude of the plasma density gradient and the driver intensity one can control the expansion rate of the blowout radius so that electron trapping occurs.

The basic idea is illustrated in Fig. 1. In the simulations used to generate Figs. $1-3$, we use $512 \times 512 \times 320$ cells in the $x, y$ and $z$ directions respectively (a longer simulation box with 416 cells in the $z$-direction is used when $\Lambda=4$ ). The cell sizes are $\frac{1}{32} \frac{c}{\omega_{p 0}}$ in each direction and 4-8 particles per cell are used for the plasma electrons (the ions are kept fixed). Here $\omega_{p 0}$ is the plasma frequency corresponding to the lower shelf density $n_{p 0}$. When a high current electron bunch propagates through plasma, a nonlinear plasma wave structure can be excited if the bunch peak density $n_{b}$ exceeds the plasma density $n_{p}$ [32-34] and the peak normalized charge per unit length, $\Lambda \equiv 4 \pi r_{e} \int_{0}^{r \gg \sigma_{r}} d r r_{b}$ exceeds unity, where $\sigma_{r}$ is the spot size of the beam and $r_{e}$ is the classical electron radius. For $\Lambda \gg 1$, the Coulomb force of the drive electron bunch "blows out" the plasma electrons which then form a thin sheath surrounding a "bubble"-like region that contains only the "immobile" ions. In the laser driver case, a similar bubble structure is

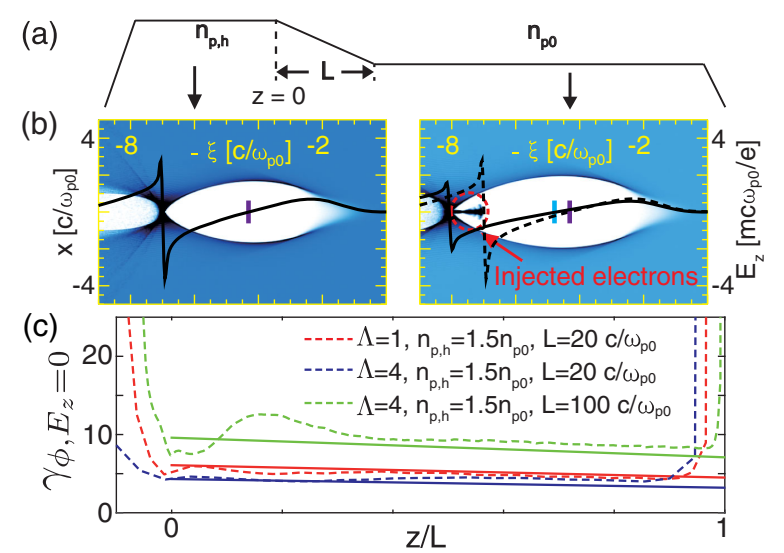

FIG. 1. (a) Schematic of density downramp injection. The plasma density decreases linearly from $n_{p, h}$ at $z=0$ to $n_{p 0}$ at $z=L$. (b) The plasma wake produced by a short electron bunch with $\Lambda=1$ before (left) and after (right) it propagates through the density downramp. The black lines are the on-axis $E_{z}$ and the purple (blue) marker indicates the position where $E_{z}=0$ when the beam is before (after) the ramp. (c) Evolution of the phase velocity $\gamma_{\phi, E_{z}=0}$ from Eq. (1) (solid lines) and 3D PIC simulations (dashed lines). The parameters are: $\gamma_{b}=2500$, $n_{b}=16 n_{p 0}, \sigma_{z}=0.7 \frac{c}{\omega_{p 0}}, \sigma_{r}=0.25 \frac{c}{\omega_{p 0}}$ when $\Lambda=1$ and $\sigma_{r}=$ $0.5 \frac{c}{\omega_{p 0}}$ when $\Lambda=4$.

formed if the normalized vector potential $a_{0} \equiv \frac{e A_{0}}{m c^{2}} \gg 1$ where $A_{0}$ is the peak vector potential of the laser [33-36]. The blowout or bubble regime has many beneficial properties for an accelerating structure; it has an ultrahigh accelerating field $E_{z}$ for electrons that is independent of the radial position and it has a large focusing field that is linear in $r$ and independent of the phase of the wake $[33,34]$.

In the blowout regime, the edge of the ion column is called the blowout radius, $r_{b}(\xi)$ (the radius is in cylindrical coordinates for each value of $\xi$ ) where $\xi \equiv v_{d} t-z \approx c t-z$. The maximum value of $r_{b}$ is defined as $r_{m}$ which for a particle beam driver is $r_{m} \approx 2 \sqrt{\Lambda} c / \omega_{p}$ [33,34]. When $r_{m} \gg c / \omega_{p}$ then $r_{b}(\xi)$ nearly maps out a circle so the wake resembles a spherical bubble. The wavelength of the wake is therefore $\lambda_{\text {wake }} \approx 2 r_{m} \approx 4 \sqrt{\Lambda} c / \omega_{p}$. The nonlinear frequency is $\omega_{N L}=\frac{\pi \omega_{p}}{2 \sqrt{\Lambda}}$. Therefore, $\omega_{p}$ can be replaced by $\omega_{N L}$ in the expression for the phase velocity. For the velocity of the first density spike, we can replace $\left(v_{d} t-z\right)$ with $\lambda_{\text {wake }}$ in Eq. (1) leading to $v_{\phi} \approx v_{d}\left(1-4 \sqrt{\Lambda} \frac{c \mathrm{~d} \omega_{p}^{-1}}{\mathrm{~d} z}\right)$ and hence $\gamma_{\phi}(z, t) \equiv\left(1-\frac{v_{\phi}^{2}}{c^{2}}\right)^{-\frac{1}{2}} \approx\left(8 \sqrt{\Lambda} \frac{c \mathrm{~d} \omega_{p}^{-1}}{\mathrm{~d} z}\right)^{-\frac{1}{2}}$. This formula indicates that the phase velocity is insensitive to the exact density profile of the ramp, thus linear profiles are used in this paper for simplicity. Other profiles with similar density scale-lengths $\left(l \equiv\left|\frac{n_{p}}{\mathrm{~d} n_{p} / \mathrm{d} z}\right|\right)$ will also work.

To obtain $v_{\phi}$ from simulations, we track where $E_{z}=0$ because its location is well defined and assume it behaves 
similarly to the location of the density spike where $E_{z}$ is a minimum. In Fig. 1(c) we plot $\gamma_{\phi, E_{z}=0}$ for several values of $L$ and $\Lambda$ and reasonable agreement between theory [we use $r_{m}=\lambda_{\text {wake }} / 2$ for $\left(v_{d} t-z\right)$ in Eq. (1) to get $\left.\gamma_{\phi, E_{z}=0} \approx\left(4 \sqrt{\Lambda} \frac{c \mathrm{~d} \omega_{p}^{-1}}{\mathrm{~d} z}\right)^{-\frac{1}{2}}\right]$ and simulations is seen.

If the density of the ramp decreases gradually, i.e., $l \equiv\left|\frac{n_{p}}{\mathrm{~d} n_{p} / \mathrm{d} z}\right| \gg \frac{c}{\omega_{p}}$, the motion of an electron before injection is similar to its motion in a uniform plasma. As pointed out in Refs. [33,34], in the blowout regime the trajectories of plasma electrons vary significantly depending on their impact parameter, i.e., the initial radius, $r_{i}$. As seen in Fig. 2(a), electrons with small $r_{i} \ll r_{m}$ are deflected by the driver whereas the electrons with large $r_{i} \gg r_{m}$ are hardly perturbed. Only the electrons with $r_{i} \approx \kappa r_{m}$ form the high density narrow sheath of the wake, where $\kappa \approx \frac{1}{2}$ and its precise value depends on the intensity and profile of the driver, and can be deduced from simulations [33,34]. These electrons obtain large longitudinal forward velocity $\gamma_{z} \equiv$ $\left(1-\beta_{z}^{2}\right)^{-\frac{1}{2}}$ when they reach the rear of the wake as shown by the dashed lines (curves of $-\gamma_{z}$ vs $\xi$ for different $r_{i}$ ) in Fig. 2(a). The dependence of the maximum $\gamma_{z, M}$ on $r_{i}$ for different driver intensities $(\Lambda=1$ and 4$)$ are shown in the inset in Fig. 2(a). One can see the stronger driver generates electrons with larger $\gamma_{z, M}$. Examining Figs. 1(c) and 2(a) one sees that when the driver propagates through a gradual density downramp, $\gamma_{z, M} \geq \gamma_{\phi}$ for some sheath electrons as they are pulled back to the axis which leads to injection. This selection mechanism determines the beam quality generated in density downramp injection.

It is now well understood that in the blowout regime [see Fig. 2(a)], electrons are expelled forming a narrow sheath
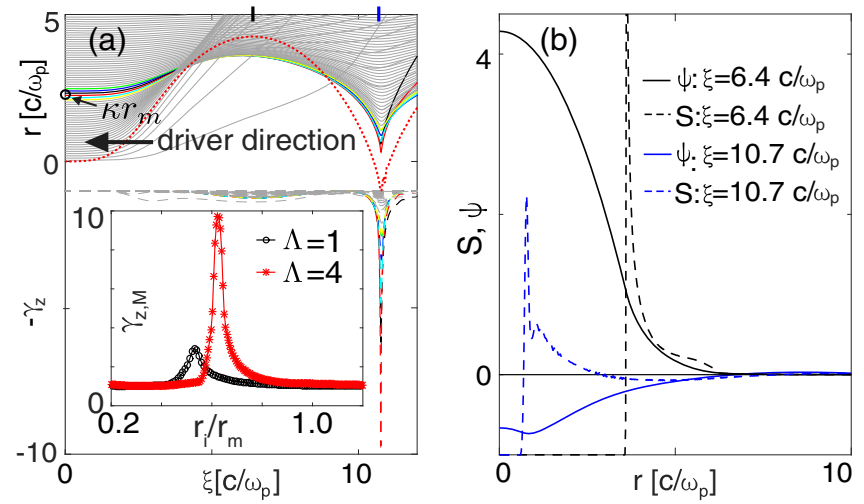

FIG. 2. (a) The trajectories (solid lines) and the $-\gamma_{z}$ (dashed lines) of the electrons in a uniform plasma with $n_{p}=1.5 n_{p 0}$ and $\omega_{p}$ is the corresponding plasma frequency. The red dotted line is the on-axis $\psi_{0}$. The inset shows the dependence of $\gamma_{z, M}$ of the electrons on the initial radius under different drivers. The black and blue markers correspond to $\xi=6.4 c / \omega_{p}$ and $10.7 c / \omega_{p}$. (b) The source term $S$ and pseudo-potential $\psi$ at different $\xi$. The blue dashed line is an average. The parameters of the beam drivers are the same as the $\Lambda=4$ case in Fig. 1 . and that the ion column provides a restoring force which pulls these electrons back toward the $r=0$ axis. As the electrons return to the axis their axial velocity begins to approach the speed of light and their radial momentum becomes large and negative. This indicates that the transverse emittance would be large if these electrons are trapped. However, a more detailed investigation reveals that wakefield excitation and the resulting dynamics of these electrons are more complicated. A quick inspection [Fig. 2(a)] demonstrates that the electrons in the sheath actually do not across the axis (a very small number of the sheath electrons across the axis but the majority do not); rather, they are reflected before reaching the axis indicating the transverse momentum $p_{\perp}$ vanishes as they approach the axis.

In a uniform plasma and assuming the phase velocity of the wake is near the speed of light, the longitudinal velocity of the electron can be approximately described as $\beta_{z}=1-\frac{2(1+\psi)^{2}}{1+\left(p_{\perp} / m c\right)^{2}+(1+\psi)^{2}}$ [37], where $\psi \equiv \frac{e}{m c^{2}}\left(\phi-A_{z}\right)$ is the wake potential and $\phi$ and $A_{z}$ are the scalar potential and the axial component of the vector potential, respectively. This expression shows that either $p_{\perp}$ goes to infinity and/or $\psi$ must approach -1 in order for $\beta_{z}$ to approach unity. As shown below, $p_{\perp}$ remains finite so $\psi$ must approach -1 . In fact a general trapping condition can be derived without assuming $\gamma_{\phi} \rightarrow \infty$ as $\psi \leq-1+\frac{\sqrt{1+\left(p_{\perp} / m c\right)^{2}}}{\gamma_{\phi}}$ [14].

The wake potential $\psi$ obeys the Poisson's equation [33]

$$
\nabla_{\perp}^{2} \psi=S \equiv-\frac{1}{n_{p} e}\left(\rho-\frac{J_{z}}{c}\right)
$$

where $S=-1$ inside the ion column. Lu et al. showed for a simple phenomenological model of a sheath with a single width, $\Delta$, where $S \geq 0$ outside the ion column ( $\mathrm{S}$ is constant and greater than unity in the sheath and 0 outside the sheath), that $\psi$ inside the ion column takes the general form $\psi=\frac{r_{b}^{2}(\xi)}{4}\left(1+\beta_{\Delta}\right)-\frac{r^{2}}{4} \equiv \psi_{0}-\frac{r^{2}}{4}$, where $\beta_{\Delta}$ is a positive definite function. Therefore for such a sheath, $\psi \geq 0$ at each transverse position, such as in the case for the black lines in Fig. 2(b). In fact it can be shown $\psi \geq 0$ for any solution of Eq. (2) where $S>0$ outside the ion column. However we find that at the very rear of the wake, $S<0$ for some $r$ outside the ion column such as in the case for the blue lines in Fig. 2(b). As a result $\psi$ can be very close to -1 at the rear of the wake and this is why the electrons can be trapped.

Very importantly, we have also found that in the rear of the wake, $\left|p_{\perp}\right|$ vanishes for almost all sheath electrons as the approach the $r=0$ axis. This is true for wake excitation in both uniform density plasmas and in density downramps. As a result electrons are reflected before they reach the axis. The electrons that are actually trapped also behave similarly as they approach the axis which is one key reason for why such low emittances are possible. 
To understand why, we look more closely at the transverse dynamics of electrons at the edge of the sheath. The transverse force, $-e\left(\vec{E}+\frac{\vec{v}}{c} \times \vec{B}\right)_{\perp}$, on a plasma electron at $r=r_{b}(\xi)$ can be written as $F_{r}=F_{d}+F_{i}+F_{e}$ [33,34], where $F_{d}=m c \omega_{p}\left(1-\beta_{z}\right) \frac{\Lambda(\xi)}{k_{p} r}$ is from the beam driver, $F_{i}=-m c \omega_{p} \frac{k_{p} r}{2}$ is from the ion cavity and $F_{e}=-m c \omega_{p} \frac{k_{p} r}{2}\left(1-\beta_{z}\right) \frac{\mathrm{d}^{2} \psi_{0}}{\mathrm{~d}\left(k_{p} \xi\right)^{2}}=-m c \omega_{p} \frac{k_{p} r}{2}\left(1-\beta_{z}\right) \frac{\mathrm{d}\left(\frac{e E_{z}}{m c \omega_{p}}\right)}{\mathrm{d}\left(k_{p} \xi\right)}$ is from the radial current of the plasma electrons.

Initially, the defocusing force (D) from the driver $F_{d}$ dominates-this is what expels the plasma electrons outward forming the narrow sheath. As the driver passes by the electrons, the transverse force is solely due to $F_{i}+F_{e}$. For most of the wake, $\frac{\mathrm{d}^{2} \psi_{0}}{\mathrm{~d}\left(k_{p} \xi\right)^{2}} \approx-\frac{1}{2}[33,34]$, thus $\frac{F_{i}+F_{e}}{r} \approx$ $-\frac{m \omega_{p}^{2}}{4}\left(1+\beta_{z}\right)<0$ which is focusing $(\mathrm{F})$ and the plasma electrons are pulled back to the ions. However, at the very rear of the wake $(\xi>10$ for the $\Lambda=4$ case shown in Figs. 2 and 3), the $F_{e}$ can dominate leading to $\frac{F_{r}}{r}>0$ [see Fig. 3(a)]. As shown in Fig. 2(a), $\gamma_{z}$ is $O(10)$ so that $1-\beta_{z}$ is $O(.01)$. However, $\left|\frac{\mathrm{d}^{2} \psi_{0}}{\mathrm{~d}\left(k_{p} \xi\right)^{2}}\right|=\left|\frac{\mathrm{d}\left(\frac{e E_{z}}{c \omega_{p}}\right)}{\mathrm{d}\left(k_{p} \xi\right)}\right|$ can easily exceed 100 in nonlinear wakes. As a result electrons are decelerated in the transverse direction as they rush back to the axis and they are reflected before they reach the axis for a uniform plasma. This is seen in Fig. 3(b) [and in Fig. 2(a)] where the trajectories in $\left(x, p_{x}\right)$ phase space are shown for sample electrons who start at different radii (different initial $x$ but with initial $y=0$ ). Solid lines are for a uniform plasma and the dashed line shows how the trajectory for a
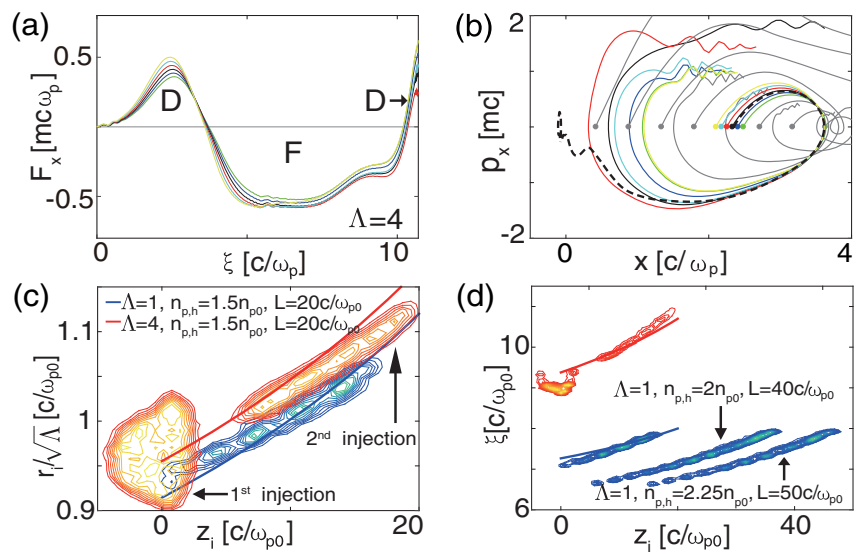

(d)

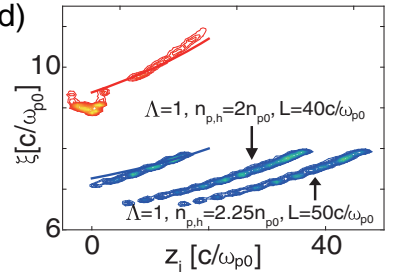

FIG. 3. (a) The transverse forces experienced by a sample of the sheath electrons with initial positive $x_{i}$. (b) The trajectories of the electrons in the transverse phase space. The initial positions $(x=$ $x_{i}$ ) are indicated by dots. The solid lines are for a uniform plasma (no injection) while the dashed line is for a sample injected electron in a downramp. The electrons in (a) and (b) have $y_{i} \approx 0$ and the same colors correspond to the same electrons. The normalized charge density distribution of the injected electrons in $r_{i}-z_{i}$ plane (c) and $\xi-z_{i}$ plane (d). The lines are from Eq. (3). The beam parameters are the same as in Fig. 1. typical trapped electron in a density gradient. We studied this deceleration process using even finer resolution (finer than $\frac{1}{32} \frac{c}{\omega_{p 0}}$ ) and found that although the axial profiles of the on-axis $\psi_{0}$ depend on the resolution, the deceleration process always exists and the emittance of the downramp injected electrons are similar.

It can be seen the electrons which start near $x_{i} \approx$ $2 c / \omega_{p}=\sqrt{\Lambda} c / \omega_{p}$ are the ones which form the sheath and approach the $x=0$. As this occurs these electrons have $\beta_{z} \sim 1$. As noted above although the $\left(1-\beta_{z}\right)$ term becomes very small, the transverse force, $F_{r}$ becomes positive (defocusing) and they remain in phase with the defocusing fields so that the total impulse from $F_{r}$ is significant. The net effect as seen in Fig. 3(b) is that for sheath electrons $\left|p_{\perp}\right|=\left|p_{x}\right|$ reduces to zero and then begins to increase. However, in a downramp for which the phase velocity is decreased for trapping to occur the dynamics for a trapped electron is very different. As seen by the dashed line in Fig. 3(b) the $\left|p_{\perp}\right|$ for such an electron is found to decrease to a very small value and then remain small. The reason for this is that before the electron can be reflected it moves forward relative to the tail of the wake due to the further expansion of the wake. It thus moves from the region near the density spike where there is a large transverse deceleration (defocusing) field, into region where it sees the standard linear focusing force from the ion column. At this point it conducts the transverse betatron motion under the ion focusing force with a very small excursion in $x_{\perp}$ and in $p_{\perp}$.

As just noted, after injection the electrons gradually move forward relative to the rear of the wake due to the further expansion of the wake, i.e., they do not phase slip with respect to the driver but they do with the rear of the bubble. Meanwhile, $r_{i}$ (in absolute units) of the injected electrons increases because it depends on density. There is also a one-to-one mapping between $\xi$ (the axial location of the electron after the downramp region) and the initial longitudinal position $z_{i}$, which eliminates longitudinal phase mixing [17], i.e., the electrons in a final, $\xi$ slice originate from the same $z_{i}$, leading to a small slice energy spread. The dependence of $r_{i}$ and $\xi$ on $z_{i}$ are

$$
\frac{\mathrm{d} r_{i}}{\mathrm{~d} z_{i}} \approx \kappa \frac{\mathrm{d} r_{m}}{\mathrm{~d} z_{i}}, \quad \frac{\mathrm{d} \xi}{\mathrm{d} z_{i}} \approx \frac{\mathrm{d} \lambda_{\text {wake }}}{\mathrm{d} z_{i}} \approx 2 \frac{\mathrm{d} r_{m}}{\mathrm{~d} z_{i}}
$$

where $\kappa$ depends weakly on $z_{i}$. In Figs. 3(c) and 3(d), the dependence of $r_{i}$ and $\xi$ on $z_{i}$ for the injected electrons with different parameters are shown. Good agreement between simulation results and Eq. (2) is found. From the correlation between $\xi$ and $z_{i}$, an injected beam with the duration much less than $c / \omega_{p 0}$ is expected if a ramp with the same gradient but shorter length is used.

When $\Lambda=1$, the electrons are injected continuously as the driver propagates in the ramp. When $\Lambda=4$, there is 
significant injection around the starting point of the ramp $\left(z_{i}=0\right)$. Here the electrons from different $z_{i}$ mix together, leading to a large slice energy spread and large current. These injected electrons load the wake [38], decreasing $v_{z}$ of the subsequent electrons in the sheath and the injection ceases. As the beam propagates deeper into the low density region, $v_{\phi}$ decreases further and the electrons move forward in the wake. The beam loading from the initial injection then weakens [38], leading to a separate injection which is continuous for $\Lambda=1$. A smooth transition of the plasma density instead of a abrupt one can suppress the first violent injection and let the whole injection more uniform in this high current driver case. In Fig. 3(d), we show two more cases for $\Lambda=1$ with the same linear density gradient and $n_{p 0}$ but different $L$ (i.e., different $n_{p, h}$ ). Because $v_{\phi}$ of the wake is always higher at the beginning of the ramp [see Fig. 1(c)], then $v_{\phi}$ will cross its threshold at the same density and this is confirmed. Thus the injected beams are almost the same in these two cases $\left(L=40 c / \omega_{p 0}\right.$ and $\left.50 c / \omega_{p 0}\right)$.

The current of the injected beam can be approximated as $I \approx(e c) 2 \pi r_{i} \Delta r_{i} n_{p} \frac{\mathrm{d} z_{i}}{\mathrm{~d} \xi} \approx(2 \kappa \pi e c) \Delta r_{i} n_{p} l$, where $2 \pi r_{i} \Delta r_{i} n_{p} \mathrm{~d} z_{i}$ is the injected particle number at $z_{i}, \mathrm{~d} \xi$ is the bunch length after injection, and we use Eq. (2). The current can be controlled by adjusting the driver intensity and the gradient of the downramp. An intense driver increases both $r_{i}$ and $\Delta r_{i}$. A steeper ramp (i.e., smaller l) makes injection easier by decreasing the $\gamma_{z}$ needed for injection which then increases $\Delta r_{i}$, but it also simultaneously decreases $n_{p} l$, so it is necessary to use simulations to quantify the dependence of the current on the density scale-length.

Based on simulations, the slice emittance of the injected beam is determined by the $\Delta r_{i}$ for each slice. Large $\Delta r_{i}$ tends to generate a beam with a larger emittance. So we can fine tune the parameters of the density profile and the driver to reduce $\Delta r_{i}$ hence to reduce the emittance. The residual transverse momentum after the transverse deceleration process also affects the emittance which requires simulations to quantify. The current, slice emittance, energy spread and the brightness of the injected beam as a function of slice position are shown in Fig. 4. In Figs. 4(a) and 4(b) we can see that the emittance and the current are smaller when $\Lambda=1$ as compared to when $\Lambda=4$, this is consistent with the $\Delta r_{i}$ as shown in Fig. 3(c) where $r_{i}$ is normalized to $\sqrt{\Lambda}$. In Fig. 4(c), we can see slice energy spreads as low as $\sigma_{\gamma} \lesssim 0.1$ for the beam when $\Lambda=1$ and as low as $\sigma_{\gamma} \lesssim 0.4$ the second group of electrons when $\Lambda=4$. The slice emittance and energy spread are both an order of magnitude higher for the first group of electrons. The higher slice energy spread is consistent with the longitudinal mixing (larger range of $z_{i}$ for a $\xi$ ) for the first group of electrons seen in Fig. 3(d). There are some electrons around the core of the beam which contribute little to the current but
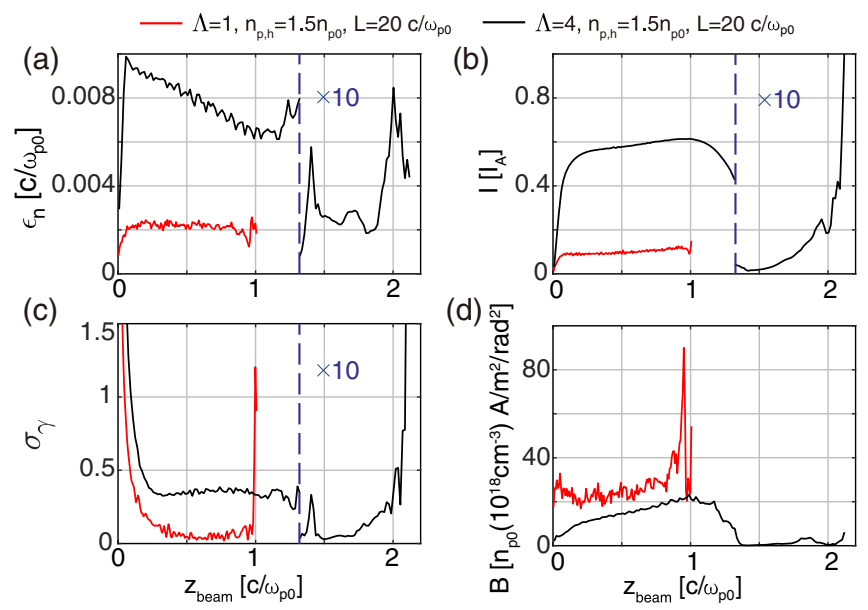

FIG. 4. The parameters of the injected beams in two conditions: (a) the slice emittance, (b) the current, (c) the slice energy spread and (d) the brightness. Here $I_{A} \approx 17 \mathrm{kA}$ is the Alfven current. The beam is divided into 128 slices. The red (black) lines corresponds to $\Lambda=1(4)$. The curves right of the blue dashed lines are attenuated by 10 .

significantly increase the emittance. We remove these particles (they can be easily filtered out in experiments) when we calculate the beam parameters. When generating the plots in Fig. 4, 80\% electrons are contained.

As we described above, the energy spread in an infinitesimally thin slice of the electron beam generated from density downramp injection can be as low as $O(0.1) \mathrm{MeV}$. Initially, the injection process generates a beam with a high energy at the head and a low energy at the tail (positive energy chirp) because the head of the beam was injected first. Thus, there is an initial chirp in momentum of the beam, $\Delta p \sim e E_{z} L / c$, where $L / c$ is the scale of the duration over which injection occurs. The head of the beam feels a smaller accelerating field than the rear so that after an optimized acceleration distance the chirp can be removed. However, the optimized acceleration distance may be significantly less than the dephasing length which limits the maximum energy gain. A longer downramp can increase the initial energy chirp thereby increasing the acceleration length (making it closer to the dephasing length) needed to remove it. There is thus a large parameter space to explore.

In reality, due to the nonlinear nature of the blowout regime (nonlinear profile of the acceleration gradient and the beam loading effect), only part of the beam can achieve a flat longitudinal phase space distribution. At the optimized distance $\left(70 c / \omega_{p 0}\right.$ after the ramp for $\Lambda=1$ and 90 $c / \omega_{p 0}$ for $\left.\Lambda=4\right)$, the projected energy spread of the flat region of the beam (about half of the beam) is about $\sigma_{\gamma} \approx 0.52(\Lambda=1), 0.33(\Lambda=4)$ with the average energy $\bar{\gamma} \approx 85.5(\Lambda=1), 116(\Lambda=4)$. Note that in order to simulate the injected beam with high fidelity and eliminate the numerical Cherenkov instabilities $[39,40]$ induced by the 
high current, relativistic injected beam, OSIRIS with either the hybrid FFT/finite-difference solver [41] or the customized finite-difference solver [42] and very fine cell sizes $\left(\frac{1}{128} \frac{c}{\omega_{p 0}}\right)$ is used in Fig. 4.

The generation of high brightness beams depends on the transverse deceleration process in the electric field spike in the tail of the wake. Plasma temperature and asymmetry of the driver can modify the wake structure (remove the spike) and therefore affect the transverse deceleration of the injected electrons. For the $\Lambda=4, n_{p, h}=1.5 n_{p 0}$, $L=20 c / \omega_{p 0}$ case, the emittance is larger by a factor 1.3 for a $40 \mathrm{eV}$ plasma electron temperature as compared with a cold plasma. However, the current and the energy spread vary little. We also find that if the aspect ratio of the driver is kept below 1.1 the beam quality is unchanged.

Each PIC simulation corresponds to an infinite set of simulations with the same normalized parameters and beam shapes, but with a different plasma density. In absolute units, the brightness of both the drive and output beam are therefore proportional to the corresponding plasma density. For the $\Lambda=1$ and 4 cases, the brightness of the output beams correspond to $\sim 30$ and $\sim 20\left(n_{p 0}\left[\mathrm{~cm}^{-3}\right]\right) \mathrm{A} / \mathrm{m}^{2} / \mathrm{rad}^{2}$ respectively. Current state-of-the-art conventional sources of electron beams can provide $\sim 10 \mathrm{kA}$ currents and pulse lengths as short as $\sim 2$ fs [43] which would allow operating in the blowout regime at densities as high as $10^{20} \mathrm{~cm}^{-3}$, indicating that unprecedented brightnesses greater than $10^{21} \mathrm{~A} / \mathrm{m}^{2} / \mathrm{rad}^{2}$ are possible.

A simulation for a laser-driver has also shown brightnesses in excess of $10^{20} \mathrm{~A} / \mathrm{m}^{2} / \mathrm{rad}^{2}$. Here we present results from an OSIRIS simulation of an $800 \mathrm{~nm}$ circularlypolarized laser pulse with $a_{0}=2 \sqrt{2}, w_{0}=5.5 \mu \mathrm{m}$ and $\tau_{\mathrm{FWHM}}=25 \mathrm{fs}$ exciting a wake in a plasma with $n_{p 0}=$ $10^{19} \mathrm{~cm}^{-3}$. The laser is focused at $z_{\text {plasma }}=-0.025 \mathrm{~mm}$, where $z_{\text {plasma }}=0 \mathrm{~mm}$ is the start of the downramp. The plasma density is decreased from $1.5 n_{p 0}$ to $n_{p 0}$ in $28 \mu \mathrm{m}$. In this example an electron beam with a peak brightness of $1.8 \times 10^{20} \mathrm{~A} / \mathrm{m}^{2} / \mathrm{rad}^{2}$ (8 kA current and $9 \mathrm{~nm}$ emittance) and $\lesssim 0.15 \mathrm{MeV}$ slice energy spread is generated as shown in Fig. 5(a). At $z_{\text {plasma }}=0.18 \mathrm{~mm}$, the projected rms energy spread of the injected beam between $z=1.2 \mu \mathrm{m}$ and $z=$ $3.0 \mu \mathrm{m}$ is only $0.27 \mathrm{MeV}$ with a mean energy $56.5 \mathrm{MeV}$ as shown in Fig. 5(b). For the OSIRIS simulation shown in Fig. 5, we use the hybrid FFT/finite-difference solver, $1600 \times 1600 \times 1400$ cells in the $x, y$ and $z$ directions respectively, cell sizes of $0.2 c / \omega_{0}$ in each directions, and 1 particle per cell are used for the plasma electrons and ions. Here $\omega_{0}$ is the laser frequency. The mobile ions are set in this simulation because the density of the injected beam can as high as $10^{22} \mathrm{~cm}^{-3}$ and we found the ions have little impact on the beam quality.

In conclusion, we have shown that when an intense particle beam or laser propagates down a density gradient
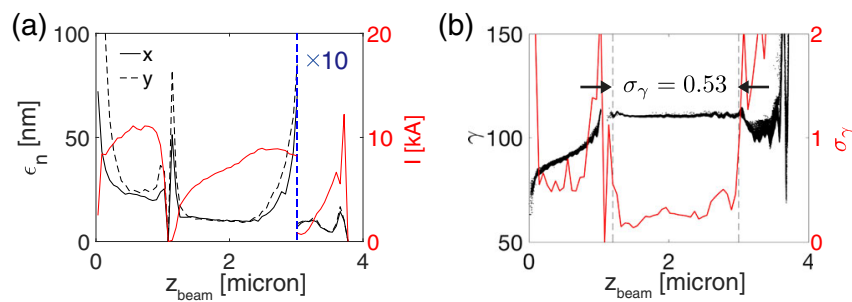

FIG. 5. The simulation results in a laser driven density ramp injection. (a) The emittance and the current at $z_{\text {plasma }}=0.18 \mathrm{~mm}$. The black lines are the emittance and the red line is the current. (b) The longitudinal phase space (black dots) and the slice energy spread (red line) at $z_{\text {plasma }}=0.18 \mathrm{~mm}$. Note that about $94 \%$ electrons are contained when calculated the beam parameters and the whole beam is divided into 64 slices.

that self-trapped electron beams with unprecedented normalized brightnesses can be produced. The scaling of this process and the underlying physics that leads to high brightness beams is studied using theory and OSIRIS simulations. The self-injected electrons originate from a narrow radial region. These electrons are initially blown outward and are pulled back to the axis by the ion column forces. However, it is shown that the high beam quality is generated due to a transverse deceleration on the electrons as they approach the axis in the tail of the wake. In a uniform plasma electrons are then reflected by this decelerated force and then move outward. However, in a density gradient, some of these electrons become trapped as they move forward in the wake with respect to the tail due to the increasing wake wavelength in the downramp. They thus leave the region of the decelerating (defocusing) field into the standard focusing field with simultaneous very small initial transverse momentum and transverse position. This leads to the generation of low emittance beams. The correlation between the final phase inside the wake and the initial longitudinal position breaks the longitudinal phase mixing and leads to a low slice energy spread. At some optimized acceleration distance, part of the beams can achieve a low projected energy spread due to the remove of the initial energy chirp along the beam. By adjusting the intensity of the driver and the gradient and the length of the ramp, beams with various duration, current, emittance and energy spread can be generated. Thus an appropriately designed density downramp injection source may lead to beams for an early application of a PBA to a next generation X-FEL and many other applications.

This work was supported by the NSFC Grants No. 11425521, No. 11535006, No. 11375006, and No. 11475101, Thousand Young Talents Program, DOE Grants No. DE-SC0010064, No. DE-SC0014260, and NSF Grants No. 1500630, No. 1734315, No. ACI-1339893, No. ACI-1614949, No. ACI-1713760. The simulations were performed on the UCLA Hoffman 2 and Dawson 2 
Clusters, and the resources of the National Energy Research Scientific Computing Center and the Blue Waters.

X. L. Xu and F. Li contributed equally to this work.

[1] C. Joshi and T. Katsouleas, Plasma accelerators at the energy frontier and on tabletops, Phys. Today 56, No. 6, 47 (2003).

[2] W. Barletta, J. Bisognano, J. Corlett, P. Emma, Z. Huang, K.-J. Kim, R. Lindberg, J. Murphy, G. Neil, D. Nguyen et al., Free electron lasers: Present status and future challenges, Nucl. Instrum. Methods Phys. Res., Sect. A 618, 69 (2010), and references therein.

[3] R. Tomás, Overview of the compact linear collider, Phys. Rev. ST Accel. Beams 13, 014801 (2010), and references therein.

[4] F. S. Tsung, R. Narang, W. B. Mori, C. Joshi, R. A. Fonseca, and L. O. Silva, Near-GeV-Energy LaserWakefield Acceleration of Self-Injected Electrons in a Centimeter-Scale Plasma Channel, Phys. Rev. Lett. 93, 185002 (2004).

[5] S. Gordienko and A. Pukhov, Scalings for ultrarelativistic laser plasmas and quasimonoenergetic electrons, Phys. Plasmas (1994-present) 12, 043109 (2005).

[6] W. Lu, M. Tzoufras, C. Joshi, F. S. Tsung, W. B. Mori, J. Vieira, R. A. Fonseca, and L. O. Silva, Generating multi$\mathrm{GeV}$ electron bunches using single stage laser wakefield acceleration in a 3D nonlinear regime, Phys. Rev. ST Accel. Beams 10, 061301 (2007).

[7] S. Kalmykov, S. A. Yi, V. Khudik, and G. Shvets, Electron Self-Injection and Trapping into an Evolving Plasma Bubble, Phys. Rev. Lett. 103, 135004 (2009).

[8] D. H. Froula et al., Measurements of the Critical Power for Self-Injection of Electrons in a Laser Wakefield Accelerator, Phys. Rev. Lett. 103, 215006 (2009).

[9] S. Mangles, C. Murphy, Z. Najmudin, A. Thomas, J. Collier, A. Dangor, E. Divall, P. Foster, J. Gallacher, C. Hooker et al., Monoenergetic beams of relativistic electrons from intense laser-plasma interactions, Nature (London) 431, 535 (2004).

[10] C. Geddes, C. Toth, J. Van Tilborg, E. Esarey, C. Schroeder, D. Bruhwiler, C. Nieter, J. Cary, and W. Leemans, High-quality electron beams from a laser wakefield accelerator using plasma-channel guiding, Nature (London) 431, 538 (2004).

[11] J. Faure, Y. Glinec, A. Pukhov, S. Kiselev, S. Gordienko, E. Lefebvre, J.-P. Rousseau, F. Burgy, and V. Malka, A laser-plasma accelerator producing monoenergetic electron beams, Nature (London) 431, 541 (2004).

[12] M. Chen, Z.-M. Sheng, Y.-Y. Ma, and J. Zhang, Electron injection and trapping in a laser wakefield by field ionization to high-charge states of gases, J. Appl. Phys. 99, 056109 (2006).

[13] E. Oz et al., Ionization-Induced Electron Trapping in Ultrarelativistic Plasma Wakes, Phys. Rev. Lett. 98, 084801 (2007).
[14] A. Pak, K. A. Marsh, S. F. Martins, W. Lu, W. B. Mori, and C. Joshi, Injection and Trapping of Tunnel-Ionized Electrons into Laser-Produced Wakes, Phys. Rev. Lett. 104, 025003 (2010).

[15] B. Hidding, G. Pretzler, J. B. Rosenzweig, T. Königstein, D. Schiller, and D. L. Bruhwiler, Ultracold Electron Bunch Generation via Plasma Photocathode Emission and Acceleration in a Beam-Driven Plasma Blowout, Phys. Rev. Lett. 108, 035001 (2012).

[16] F. Li et al., Generating High-Brightness Electron Beams via Ionization Injection by Transverse Colliding Lasers in a Plasma-Wakefield Accelerator, Phys. Rev. Lett. 111, 015003 (2013).

[17] X.L. Xu et al., Phase-Space Dynamics of Ionization Injection in Plasma-Based Accelerators, Phys. Rev. Lett. 112, 035003 (2014).

[18] D. Umstadter, J. K. Kim, and E. Dodd, Laser Injection of Ultrashort Electron Pulses into Wakefield Plasma Waves, Phys. Rev. Lett. 76, 2073 (1996).

[19] E. Esarey, R. F. Hubbard, W. P. Leemans, A. Ting, and P. Sprangle, Electron Injection into Plasma Wakefields by Colliding Laser Pulses, Phys. Rev. Lett. 79, 2682 (1997).

[20] J. Faure, C. Rechatin, A. Norlin, A. Lifschitz, Y. Glinec, and V. Malka, Controlled injection and acceleration of electrons in plasma wakefields by colliding laser pulses, Nature (London) 444, 737 (2006).

[21] X. Davoine, E. Lefebvre, C. Rechatin, J. Faure, and V. Malka, Cold Optical Injection Producing Monoenergetic, Multi-GeV Electron Bunches, Phys. Rev. Lett. 102, 065001 (2009).

[22] S. Bulanov, N. Naumova, F. Pegoraro, and J. Sakai, Particle injection into the wave acceleration phase due to nonlinear wake wave breaking, Phys. Rev. E 58, R5257 (1998).

[23] H. Suk, N. Barov, J. B. Rosenzweig, and E. Esarey, Plasma Electron Trapping and Acceleration in a Plasma Wake Field Using a Density Transition, Phys. Rev. Lett. 86, 1011 (2001).

[24] I. Kostyukov, E. Nerush, A. Pukhov, and V. Seredov, Electron Self-Injection in Multidimensional RelativisticPlasma Wake Fields, Phys. Rev. Lett. 103, 175003 (2009).

[25] R. Lehe, A. F. Lifschitz, X. Davoine, C. Thaury, and V. Malka, Optical Transverse Injection in Laser-Plasma Acceleration, Phys. Rev. Lett. 111, 085005 (2013).

[26] T. Katsouleas, Physical mechanisms in the plasma wakefield accelerator, Phys. Rev. A 33, 2056 (1986).

[27] C. G. R. Geddes, K. Nakamura, G. R. Plateau, Cs. Toth, E. Cormier-Michel, E. Esarey, C. B. Schroeder, J. R. Cary, and W. P. Leemans, Plasma-Density-Gradient Injection of Low Absolute-Momentum-Spread Electron Bunches, Phys. Rev. Lett. 100, 215004 (2008).

[28] A. Gonsalves, K. Nakamura, C. Lin, D. Panasenko, S. Shiraishi, T. Sokollik, C. Benedetti, C. Schroeder, C. Geddes, J. Van Tilborg et al., Tunable laser plasma accelerator based on longitudinal density tailoring, Nat. Phys. 7, 862 (2011).

[29] A. Buck, J. Wenz, J. Xu, K. Khrennikov, K. Schmid, M. Heigoldt, J. M. Mikhailova, M. Geissler, B. Shen, F. Krausz et al., Shock-Front Injector for High-Quality Laser-Plasma Acceleration, Phys. Rev. Lett. 110, 185006 (2013).

[30] J. Grebenyuk, A. M. de la Ossa, T. Mehrling, and J. Osterhoff, Beam-driven plasma-based acceleration of 
electrons with density down-ramp injection at FLASHForward, Nucl. Instrum. Methods Phys. Res., Sect. A 740, 246 (2014).

[31] R. Fonseca et al., OSIRIS: A three-dimensional, fully relativistic particle in cell code for modeling plasma based accelerators, Lect. Notes Comput. Sci. 2331, 342 (2002).

[32] J. B. Rosenzweig, B. Breizman, T. Katsouleas, and J. J. Su, Acceleration and focusing of electrons in two-dimensional nonlinear plasma wake fields, Phys. Rev. A 44, R6189 (1991).

[33] W. Lu, C. Huang, M. Zhou, W. B. Mori, and T. Katsouleas, Nonlinear Theory for Relativistic Plasma Wakefields in the Blowout Regime, Phys. Rev. Lett. 96, 165002 (2006).

[34] W. Lu, C. Huang, M. Zhou, M. Tzoufras, F. S. Tsung, W. B. Mori, and T. Katsouleas, A nonlinear theory for multidimensional relativistic plasma wave wakefields, Phys. Plasmas 13, 056709 (2006).

[35] W. Mori, T. Katsouleas, C. Darrow, C. Clayton, C. Joshi, J. Dawson, C. Decker, K. Marsh, and S. Wilks, Laser wakefields at UCLA and LLNL, in Proceedings of the 1991 Particle Accelerator Conference, San Francisco, CA, 1991 (IEEE, New York, 1991).

[36] A. Pukhov and J. Meyer-ter Vehn, Laser wake field acceleration: the highly non-linear broken-wave regime, Appl. Phys. B 74, 355 (2002).
[37] P. Mora and T. M. Antonsen Jr, Kinetic modeling of intense, short laser pulses propagating in tenuous plasmas, Phys. Plasmas (1994-present) 4, 217 (1997).

[38] M. Tzoufras, W. Lu, F. S. Tsung, C. Huang, W. B. Mori, T. Katsouleas, J. Vieira, R. A. Fonseca, and L. O. Silva, Beam Loading in the Nonlinear Regime of Plasma-Based Acceleration, Phys. Rev. Lett. 101, 145002 (2008).

[39] B. B. Godfrey and J.-L. Vay, Numerical stability of relativistic beam multidimensional PIC simulations employing the Esirkepov algorithm, J. Comput. Phys. 248, 33 (2013).

[40] X. Xu, P. Yu, S. F. Martins, F. S. Tsung, V. K. Decyk, J. Vieira, R. A. Fonseca, W. Lu, L. O. Silva, and W. B. Mori, Numerical instability due to relativistic plasma drift in EM-PIC simulations, Comput. Phys. Commun. 184, 2503 (2013).

[41] P. Yu, X. Xu, A. Tableman, V. K. Decyk, F. S. Tsung, F. Fiuza, A. Davidson, J. Vieira, R. A. Fonseca, W. Lu, L. O. Silva, and W. B. Mori, Comput. Phys. Commun. 197, 144 (2015).

[42] F. Li, P. Yu, X. Xu, F. Fiuza, V. K. Decyk, T. Dalichaouch, A. Davidson, A. Tableman, W. An, F. S. Tsung et al., Controlling the numerical Cerenkov instability in PIC simulations using a customized finite difference Maxwell solver and a local FFT based current correction, Comput. Phys. Commun. 214, 6 (2017).

[43] Brendan O'Shea (private communication). 